collected by a software, the Surveillance System for Nursing Workers Health -SIMOSTE in the period December 2012 to March 2013.

Results The Surveillance System for Nursing workers Health identified 1847 injuries in the period December 2012 to March 2013. There were prevalence of occurrence between nurses $(80.94 \%)$ and musculoskeletal diseases were the most prevalent $(34.70 \%)$, followed by diseases of the respiratory tract (13.05\%). Regarding the consequences, observed $10.67 \%$ of accidents and $86.63 \%$ of medical licenses.

Conclusions The data point to the need for development of new shares surveillance focused on the notification of occupational accidents and work-related diseases, and prevention of diseases. The Surveillance system is a technology capable to operationalize institutional policies regarding occupational health and the valuing of human resources, considering that monitoring and analysing the workers' health situation is an indispensible action for the planning of strategies to promote the improvement of their working conditions.

\section{ASSOCIATION BETWEEN WORK EXPOSURE, ALCOHOL INTAKE, SMOKING AND DUPUYTREN'S DISEASE IN A LARGE COHORT STUDY (GAZEL)}

${ }^{1}$ Alexis Descatha, ${ }^{1}$ Matthieu Carton, 'Zakia Mediouni, ${ }^{2}$ Christian Dumontier, ${ }^{3}{ }^{3}$ ves Roquelaure, ${ }^{1}$ Marcel Goldberg, ${ }^{1}$ Marie Zins, ${ }^{1}$ Annette Leclerc. ${ }^{1}$ UVSQ Inserm APHP, OHU U1018, Garches, Paris Area, France; ${ }^{2}$ Nice University, Nice, France; ${ }^{3}$ LUNAM University, LEEST, Angers, France

\subsection{6/oemed-2014-102362.202}

Objectives In view of the debate about biomechanical and toxic factors in Dupuytren's disease, we aimed to describe its relationship with certain occupational factors and alcohol intake and smoking.

Method Subjects in the French GAZEL cohort answered a questionnaire in 2012 included self-reported Dupuytren's disease, such as disabling Dupuytren's disease (including surgery). In 2007, self-assessed lifetime occupational biomechanical exposure was recorded (carrying loads, manipulating a vibrating tool, climbing stairs), as well as alcohol intake, smoking and diabetes mellitus. Analyses were performed on high alcohol intake, smoking and duration of relevant work exposure, stratified by gender for both outcomes.

Results A total of 13587 subjects answered the questionnaire in 2012 (73.7\% of the questionnaire sent) and constituted the sample (10 017 men and 3570 women, aged from 64 to 73 years; mean age for men 68 years and for women 65 years). Among men age, diabetes, heavy drinking and over 15 years of manipulating a vibrating tool at work were significantly associated with Dupuytren's disease; except for diabetes, the association with these factors was stronger for disabling Dupuytren's disease (or surgery). Among the 3570 women included, 160 reported Dupuytren's disease (4.5\%). The number of cases in the group of women was too low to reach conclusions, although the findings seemed similar for age, diabetes and vibration exposure.

Conclusions In this large French cohort study, Dupuytren's disease in men was associated with high levels of alcohol consumption and exposure to hand-transmitted vibration. It is likely that the same applied to women.

\section{0 \\ AN INTERNATIONAL HISTORICAL COHORT STUDY OF WORKERS IN THE HARD-METAL INDUSTRY: EXPOSURE ASSESSMENT}

${ }^{1}$ Kathleen Kennedy, ${ }^{1}$ Nurtan Esmen, ${ }^{2}$ Gary Marsh, ${ }^{2}$ Jeanine Buchanich, ${ }^{2}$ Sarah Downing Zimmerman, ${ }^{3}$ Hanns Moshammer, ${ }^{4,5}$ Peter Morfeld, ${ }^{4}$ Thomas Erren, ${ }^{6}$ Magnus Svartengren, ${ }^{7}$ Hakan Westberg, ${ }^{8}$ Damien McElvenny, ${ }^{8}$ John Cherrie. ${ }^{1}$ University of Illinois at Chicago, Division of Environmental and Occupational Health Sciences, Chicago, IL, USA; '2University of Pittsburgh, Center for Occupational Biostatistics and Epidemiology, Pittsburgh, PA, USA; ${ }^{3}$ Medical University of Vienna, Institute of Environmental Health, Vienna, Austria; ${ }^{4}$ Institute for Occupational Medicine, Environmental Medicine and Prevention Research of Cologne University, Cologne, Germany; ${ }^{5}$ Institute for Occupational Epidemiology and Risk Assessment of Evonik Industries, Essen, Germany; ${ }^{6}$ Uppsala University, Department of Medical Sciences, Uppsala, Sweden; ${ }^{7}$ Örebro University, Department of Occupational and Environmental Medicine, Örebro, Sweden; ${ }^{8}$ Institute of Occupational Medicine, Edinburgh, UK

\subsection{6/oemed-2014-102362.203}

Objectives A multinational occupational epidemiological study is underway to investigate the total and cause-specific mortality of workers exposed to tungsten carbide with a cobalt binder (WCCo). The study includes 12 US and 9 European plants. The objective of the exposure assessment component, coordinated by the University of Illinois at Chicago, is to reconstruct agent-specific exposure estimates for use in the epidemiological analyses.

Method Quantitative occupational exposures are being generated through a process of modelling and validation using industrial hygiene data from study plants. Innovative semi-quantitative methods are being developed to extrapolate data for years in which it is missing, and are based on exposure changes due to manufacturing process changes over time (e.g., moving from manual to automated methods). Company work history information is being used to construct a job dictionary. The resulting exposure estimates and job dictionary will form the job-exposure matrix.

Results The agents of interest are WCCo, tungsten carbide, tungsten, cobalt, and carbon black. Current and past working environments are being characterised according to products manufactured and operations performed and in relation to potential exposures using sampling data, job descriptions, plant and process histories, and information provided by knowledgeable plant personnel.

Conclusions Exposure estimates will be generated for the study plants. The multinational aspect of the study provides the opportunity to pool data and produce exposure estimates for all 21 facilities with potential insight into similarities or differences among countries and/or plants involved in the same global industry. This presentation will detail the progress to date on the exposure assessment effort.

\section{LEPTIN, ADIPONECTIN, AND HEART RATE VARIABILITY AMONG POLICE OFFICERS}

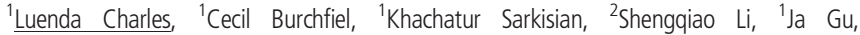
'Desta Fekedulegn, ${ }^{3}$ John Violanti, ${ }^{1}$ Michael Andrew. ${ }^{1}$ Biostatistics and Epidemiology Branch, Health Effects Laboratory Division, National Institute for Occupational Safety and Health, Centers for Disease Control and Prevention, Morgantown, West Virginia, USA; ${ }^{2}$ University of Pittsburgh Medical Center, Pittsburgh, Pennsylvannia, USA; ${ }^{3}$ Department of Social and Preventive Medicine, School of Public Health and Health Professions, State University of New York at Buffalo, Buffalo, New York, USA

\subsection{6/oemed-2014-102362.204}

Objectives To investigate the relationship of leptin and adiponectin with heart rate variability (HRV). 